\title{
Security and Privacy Protection in Datamining
}

\section{G Ravi Kumara, Dr. Harsh Pratap Singh ${ }^{\mathrm{b}}$ and Dr. N.Rajasekhar ${ }^{\mathrm{c}}$}

a Research Scholar, Dept. of Computer Science \& Engineering, Sri Satya Sai University of Technology \& Medical Sciences, Sehore, Bhopal Indore Road, Madhya Pradesh, India

${ }^{\mathbf{b}}$ Research Guide, Dept. of Computer Science \& Engineering,

Sri Satya Sai University of Technology \& Medical Sciences, Sehore, Bhopal Indore Road, Madhya Pradesh, India

${ }^{\mathbf{c}}$ Research Co-Guide,Department of Information Technology,

Gokaraju Rangaraju Institute of Engineering \& Technology (Autonomous), Hyderabad

Article History: Received: 11 January 2021; Accepted: 27 February 2021; Published online: 5 April 2021

\begin{abstract}
Security and Privacy protection have been a public approach worry for quite a long time. Notwithstanding, quick innovative changes, the fast development of the internet and electronic business, and the improvement of more modern techniques for gathering, investigating, and utilizing individual information have made privacy a significant public and government issues. The field of data mining is acquiring importance acknowledgment to the accessibility of a lot of data, effortlessly gathered and put away through PC systems. Data mining procedures, while permitting the people to remove shrouded information on one hand, present various privacy dangers then again. In this paper, we concentrate a portion of these issues alongside an itemized conversation on the utilizations of different data mining strategies for giving security. This paper gives an outline of data mining field and security information event management system. We will perceive how different data mining methods can be utilized in security information and event management system to upgrade the capacities of the system.
\end{abstract}

Keywords: Data mining, Information Security, Threats, Privacy Preserving Data Mining, Data mining, classification, clustering, intrusion.

\section{Introduction}

Data mining (DM) is the way toward extricating substantial and valuable information from huge amounts of data, dissecting the information and finding helpful examples with various procedures. It has been applied into various applications, for example, medical, health care, marketing, finance, privacy, security, etc. Security applications can be for public security to battle against psychological oppression attacks or for cyber security to ensure PCs and networks against corruption (worms and viruses), intrusion, botnet attack, malware and denial of services (DoS). No on-going methods like arrangement, forecast, and connection examination are applied to sort out a gathering of comparable dangers to decide conceivable future attacks by following viruses, while continuous procedures are more reasonable for intrusion location. Wide use of the Internet for data interchanges and sharing data over the networks increment the danger of cyber-attacks, for example, data corruption, network debasement, and unapproved admittance to classified information, etc. Because of the open idea of IP (open conventions) in $3 \mathrm{G} / 4 \mathrm{G}$ innovations, these networks are expected focuses of cyber attackers to barge in services and cause issues to the end clients and versatile administrators. The cyber-attackers could take client data, for example, IMSI number, charging information and contact subtleties, debase networks through DoS, or hinder or suspend services of a host associated with the Internet, hence making network assets inaccessible to its end clients. Data mining has developed as a decent innovation to sort out the previously mentioned security dangers. Numerous investigations have been led in the writing to identify security issues, openings, intrusions, malware, and so on in this paper; we give a careful audit on the current procedures about security related data mining, i.e., utilizing data mining strategies to sort out security issues. In this, we centre around data mining methods all in all and data mining for security applications specifically.

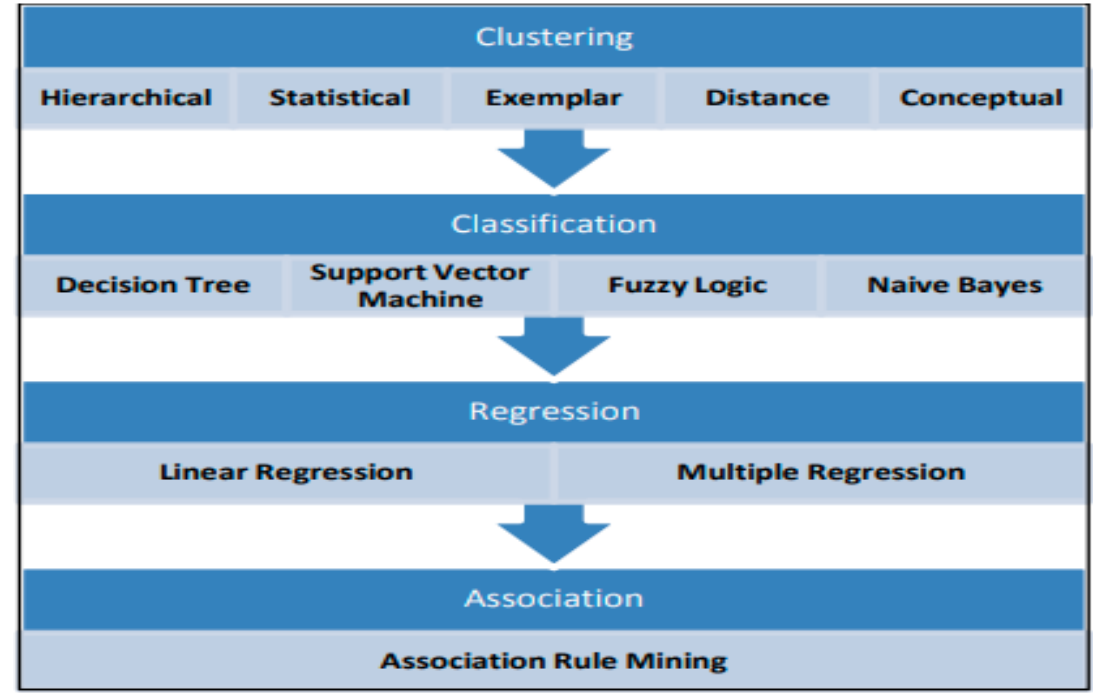

Figure 1.1 Data Mining Techniques 
Data mining (the examination step of the "Knowledge Discovery in Databases" cycle, or KDD), is a field of software engineering, which includes finding designs from enormous data sets through techniques for computerized reasoning, AI, measurements, and database systems. The primary point of the data mining measure is to extricate information from a data set and change it into a reasonable arrangement for some time later. Aside from essential investigation, the data mining measure covers database and data management viewpoints, data pre-handling, deduction contemplations, multifaceted nature contemplations, post-preparing of found designs, and web based refreshing. Foundations of Data Mining are insights, Artificial Intelligence and Machine Learning, Databases, Pattern discovery, representation, business Intelligence and so on The different Data mining strategies are recorded underneath.

- Clustering - It is the assignment of finding gatherings and designs in the data that are here and there or another "comparative", without utilizing known constructions in the data.

- Classification - It is the errand of summing up known construction which can be applied to new data. For instance, an email program may endeavour to group an email as certifiable or spam. Standard calculations are choice tree learning, Naive Bayesian arrangement, neural networks (delicate registering) and backing vector machines.

- Regression - Attempts to find a function which models the data with the least error.

- Association Rule Learning - Searches for relationships between variables.

Data mining gets its name from the likenesses between looking for important information in a huge database and mining a mountain for a vein of significant metal. The two cycles require either filtering through an enormous measure of material, or cleverly testing it to discover where the worth dwells.

\section{Role of Data Mining In Information Security}

Data mining is extraction of covered up, valuable and valuable information from enormous databases. Data mining appeared with a goal to help huge databases that are utilized in different business applications for anticipating future patterns, breaking down data and settling on proactive choices. Data mining has arisen as a device that gives its clients to distinguish the weaknesses and helps in giving a guarded instrument against various dangers to the information systems. There are different uses of data mining in the zone of information security. Generally talked about space in the field of information security is intrusion identification where the dangers to the system are distinguished and prevented. Great measure of work has been done around there by the analysts and different data mining strategies have been applied for location and prevention of security attacks on the system. With the progressions in the zone of information security, the uses of data mining has additionally expanded massively to different territories of information security and are not limited to simply intrusion recognition and prevention systems. Organization intrusion discovery is another territory which requires prompt considerations, as the quantity of intrusion attacks are expanding. It is an exceptional type of PC created danger investigation to recognize frightful activities that could bargain the trustworthiness, privacy, and accessibility of information assets. Intrusion identification components dependent on data mining are amazingly helpful in finding security penetrates. In writing, various data mining based calculations have been proposed to manage the information security and privacy issues, by utilizing approaches like order, continuous example mining, and bunching strategies to do intrusion recognition, irregularity identification, and privacy protecting. Use of these data mining techniques have brought about animating outcomes that has concerned numerous scientists in both data mining and information security regions.

\section{Literature Review}

Mehrnoosh Monshizadeh et al (2014),Data mining is the cycle that separates orders and examines legitimate and helpful information from enormous volumes of data given by numerous sources. The data mining has been generally applied into different territories, one of which is to research potential security dangers. In the writing, different data mining procedures, for example, arrangement and grouping have been proposed to recognize intrusions, DoS attacks, and malware. This paper overviews distinctive data mining methods applied to recognize security dangers and breaks down their points of interest and drawbacks. Through correlation, we examine open examination issues about security-related data mining and propose future exploration center.

MOHAMED HAMDY et al (2016), Data mining (DM) in cyber security is the way toward presenting inquiries and separating designs. In this exploration we center on an assortment of strategies, approaches and various territories of data mining structures in Cyber Security from alternate points of view, to build up an arrangement and classification that mirror the significant rules of the data mining systems in the condition of craftsmanship. Building up such a characterization impacts profoundly on directing data mining applications towards better tasks and execution as needs be Moreover we examine different sorts of cyber-attacks and how data mining can help in identification and prevention of these attacks. Information security infringement, for example, access control infringement just as a conversation of different dangers is introduced. At last we present a near investigation between a bunches of chosen structures. 


\section{EXPERIMENTAL METHODS}

Data Mining in Cyber Security is the way toward presenting questions and removing designs, regularly beforehand obscure from enormous identified with security issues. Cyber security is the region that manages shielding from cyber psychological oppression. Cyber-attacks incorporate access control infringement. Notwithstanding enhancing data mining and web mining procedures and adjusting them for counterpsychological warfare, they should share the data just as mine the data cooperatively. Here we are examining approaches to screen the enemies, for such checking to be powerful and the screen should stay away from recognition by the static and dynamic investigations utilized by standard enemy of malware bundles for creating methods that can progressively adjust to new location methodologies and keep on observing the foe. Information security is the act of shielding information from unapproved client, revelation, disturbance, adjustment or decimation. PC and correspondence systems over and over endure security and privacy attacks. These days, the majority of the organizations spend great measure of cash on their organization security and privacy prerequisites. Four key highlights of information security are referenced in figure 1.2.

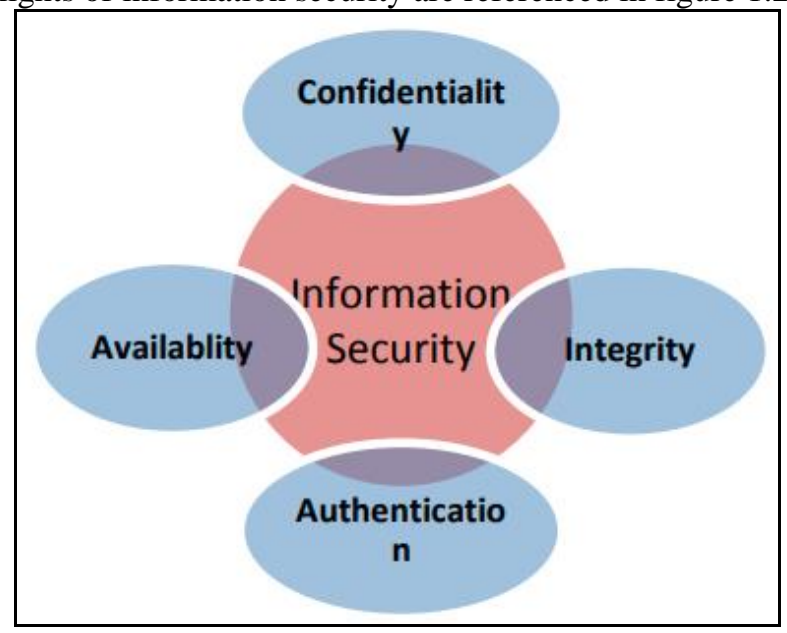

Figure 1.2 Information Security Attributes

Information security technology is a fundamental part for ensuring public and private figuring foundations. Headway in technology is making individuals more situated towards incessant utilization of information technology bringing about more use of online assets which thus is offering ascend to an enormous number of security dangers to these assets. The expanding number of security breaks is requiring some security organizations to send security arrangements and instruments to restrict or clear out these dangers.

Cyber Security

One of the fundamental prerequisites of cyber security is to give information security whose key credits are confidentiality, data integrity, authentication and data availability. Cryptography is quite possibly the most widely recognized strategies used to give security services. The initial phase toward securing a PC or organization is to perceive the dangers and gotten comfortable with the phrasings related with them. For instance, a rundown of elements or hosts that are obstructed or denied advantages or access (Blacklisted) can be distinguished. Additionally, arrangements of substances that are viewed as reliable and are conceded admittance or advantages are called White-recorded. There are essential utilities accessible with the assistance of which the cyber-attacks can be identified:

- Cryptography-The information is secured by changing over it into a disjointed arrangement (cipher text). This message can be unravelled by just the individuals who have the secret key.

- Intrusion Detection - the strategy for investigating information from networks and information systems to decide whether a security penetrate or security infringement has happened.

- Penetration Testing - an evaluation methodology whereby assessors search for vulnerabilities of a network or information system.

Data mining based cyber-attack identification includes five general stages, as outlined in Figure 1.3, that is, system observing and data catching by means of different sensors, organization/system/measure logging and sniffing daemons/specialists, and security gadgets, data pre-preparing (e.g., purifying, separating, standardization, and so on) at nearby data stores, event relationship and highlight extraction (e.g., through huge data handling, Hadoop Distributed File System (HDFS) and MapReduce), data mining (dimensionality decrease, arrangement, grouping) to recognize abuse or abnormality, perceptions and translation of mining results. Data mining based discovery, when appropriately designed, has the ability to become focal sensory system of organization. Data mining based recognition can give some valuable determined capacities, for instance, ongoing observing and episode management for security related events which are gathered from network, security gadgets, system, applications. It gives a work process which assists with following and raises the occurrence. It 
can likewise be utilized as log management, log union, and produces announcing for consistence reason. All in all, data mining based recognition thinks of an extensive range of individual parts. Cyber-crime is spread over the total cyber space which is characterized as an organization that incorporates the Internet as a significant part.

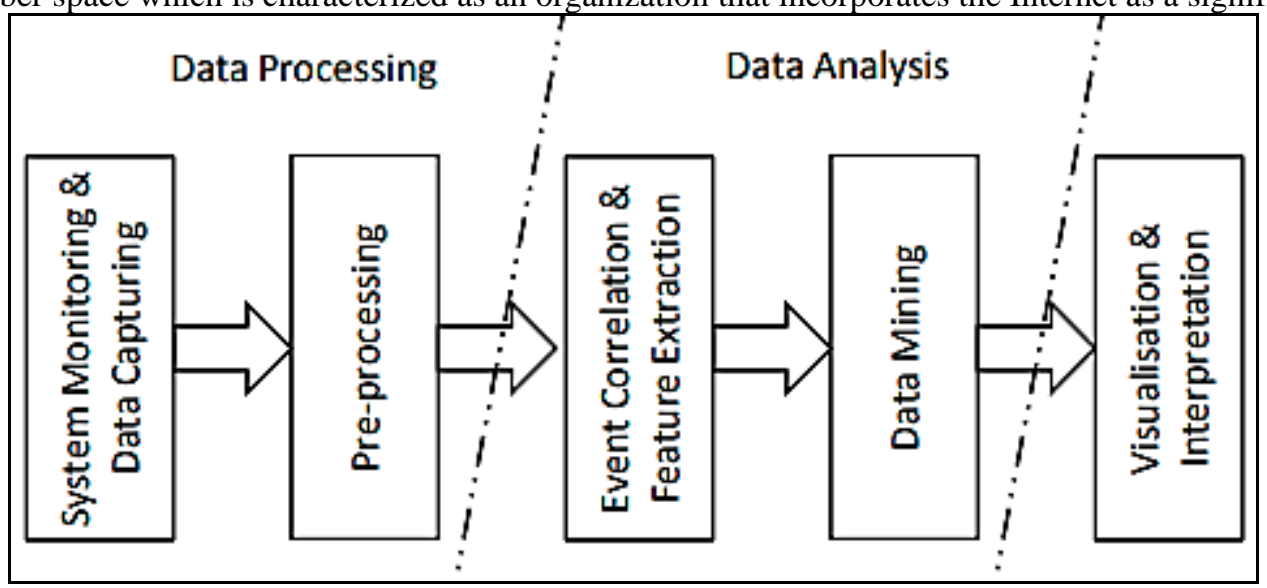

Figure 1.3 General stages in data mining based cyber-attack detection

One of the basic elements of cyber-crime is the malignant code, for example, viruses, worms, and Trojan ponies. Dynamic Attack is a purposeful danger that endeavours to change a system, its assets, its data or its tasks while inactive attack is additionally a danger that endeavours to learn or utilize information from a system however doesn't endeavour to adjust the system, its assets, its data or its activities.

[1] Viruses - This is a malignant code that requires the end client to play out some activity before it contaminates the PC like opening an email connection or going to a specific website page.

[2] Worms - Worms proliferate without client intercession and start by abusing programming weakness. Like viruses, worms can spread through email, sites, or organization based programming. The key quality of worm is that it proliferates consequently.

[3] Trojan horses - A Trojan horse program is software that does not let the user know its actual consequences. For example, a program which claims that it will speed up your computer may actually be sending confidential information to a remote intruder.

[4] Hacker, Attacker, Intruder, or Denial of Service - These terms are applied to the people who seek to exploit weaknesses in software and computer systems for their own gain. Although it is difficult to comment on one's intention for doing this because they may or may not cause direct harm to the end user but denial of service definitely deprives the end user to be properly served.

\section{Privacy Preserving Data Mining (PPDM)}

Privacy Preserving Data Mining methods focus on the extraction of important information from enormous volumes of data while securing any touchy information present in it. It guarantees the protection of touchy data to ration privacy and as yet permitting us to play out all data mining activities productively. The two sorts of privacy concerned data mining procedures are: Data privacy and Information privacy. Data privacy centres around the adjustment of the database for the protection of touchy data of the people while Information privacy centres around the change for the protection of delicate information that can be derived from the database. Then again we can say that Data privacy is worried about giving privacy to the info while Information privacy then again is tied in with giving privacy to the yield. Saving individual information from disclosure is the fundamental focal point of a PPDM calculation. The PPDM calculations depend on dissecting the mining calculations for any results that are obtained during Data privacy. The target of Privacy Preserving Data Mining is building calculations that change the first data in some way, so both the private data and information are not uncovered even after an effective mining measure. Just when some applicable satisfactory advantage is found coming about because of the entrance, the privacy laws would permit the entrance. Various gatherings may once in a while wish to share private data coming about after a fruitful accumulation without uncovering any touchy information from their end. Consider for instance, unique Book stores with separate deals data that is in a manner viewed as profoundly delicate, may wish to trade halfway information among themselves to show up at the total patterns without uncovering their individual store patterns. This requires the utilization of secure conventions for sharing the information across various gatherings. Privacy in such cases ought to be accomplished with undeniable degrees of precision. The data mining technology by guideline is impartial as far as privacy. The intention wherein a data mining calculation is utilized could either be acceptable or vindictive. Data mining has extended the examination prospects to empower analysts to abuse huge datasets on one hand, while the malignant utilization of these methods then again has presented dangers of genuine nature against protection of privacy.

Cloud Security Using in Data Mining 
Distributed computing isn't a technology yet a help which can be made accessible on interest through internet. In this day and age where individuals are searching for services like framework, programming, stage and so on advantageously, quick and easily, a cloud gives the best arrangement. Thus, client pays just for the measure of administration utilized and the span for which the help is utilized along these lines lessening the utilization, establishment and support cost. The National Institute of Standards and Technology (NIST) specifies the fundamental qualities of distributed computing as asset pooling, on-request administration, wide organization access, estimated administration, and fast flexibility. Four organization models for cloud engineering are depicted beneath:

- Private cloud: The cloud foundation is worked for a private association. It is by and large overseen by an association or an outsider.

- Community cloud: The cloud foundation is shared by a few associations and supports a particular local area that has common concerns (e.g., security necessities, strategy, and consistence contemplations). It is again overseen by an outsider or an association and may exist inside or outside the premises.

- Public cloud: The kind of cloud framework is unveiled accessible to the general or an enormous industry gathering and is claimed by an association selling cloud services.

- Hybrid cloud: The cloud framework is a synthesis of at least two mists (private, local area, or public) that stay interesting and autonomous substances, yet are bound together by some normalized or exclusive technology, which can empower movability of utilization and data.

In cloud computing, the available service models are:

Infrastructure as a Service (IaaS):It furnishes the shopper with the possibility to specify handling, stockpiling, and other crucial processing assets, and permits the purchaser to send and run programming, which may incorporate working systems and different applications. The design of cloud is appeared in figure.

Platform as a Service (PaaS):It furnishes the customer with the ability to convey onto the cloud framework; purchaser made or gained applications, delivered utilizing programming dialects and devices upheld by the supplier. The buyer has coordinate the conveyed applications just doesn't direct or run the hidden foundation like workers, organization, working systems, or capacity, and so on.

Software as a Service (SaaS):It furnishes the buyer with the ability to utilize the supplier's applications running on a cloud foundation. These applications are accessible from various customer gadgets, through interface, similar to internet browser. Like PaaS, the client has no privilege to oversee or structure the fundamental cloud framework.

Another part of security centres around virtualization. Because of the mind boggling nature of cloud, it is hard to accomplish start to finish security in a cloud additionally the limit in a cloud is recognized to be fluffy in nature. Aside from information affirmation, it is pointed that a noxious client ought to be obstructed from entering the system or whenever entered, ought to be promptly recognized and countermeasure is taken against them. A Cloud is an application stage that utilizes internet-based services to help business measure or at the end of the day, it gives a system which can be utilized to lease IT-services on a utility-like premise. The critical credits of a cloud which makes it so mainstream are: the low beginning up expenses, quick sending, costs dependent on use, and multi-occupant sharing of services. The fundamental attributes of cloud are, on interest self-administration, inescapable organization access, area autonomous asset pooling, quick flexibility, estimated administration.

\section{Conclusion}

Data mining has gotten one of the critical highlights of numerous country security activities. Regularly utilized as methods for distinguishing misrepresentation, surveying danger, and item retailing, data mining includes the utilization of data examination instruments to find beforehand obscure, legitimate examples and connections in enormous data sets. This paper gives the audit of writing on how data mining strategies and related calculations can assume an indispensable part in guaranteeing information security in a cloud. With the developing reliance of people on machines, it is needed to make a superior system to give a protected electronic-framework to work upon and guarantee information security. Cloud proposes services on interest at a much moderate rate with least overheads in this manner expanding the prominence of cloud. Simultaneously issues of information security become basic like just an approved client ought to be permitted to utilize the services of a cloud. Accordingly, need of great importance is to actualize information security in such a way that the legitimate clients get the most extreme availability of services and the invalid ones be distinguished, and halted from abusing and upsetting the services. Data mining calculations give an answer for this test of recognizing and dodging the information security attacks like intrusion, misrepresentation, information spillage, and so on this paper gives an audit of different data mining approaches which can shield a cloud from various information security attacks.

\section{REFERENCES}

[1] Amanpreet Chauhan, Gaurav Mishra, and Gulshan Kumar, "Survey on Data Mining Techniques in Intrusion Detection”, International Journal of Scientific \& Engineering Research Volume 2, Issue 7, July-2011 
[2] Han, J. and Kamber, M., "Data mining: Concepts and Techniques", Morgan-Kaufman Series of Data Management Systems. San Diego: Academic Press, 2011.

[3]I. K. R. Rao, "Data Mining and Clustering Techniques," DRTC Workshop on Semantic Web, DRTC, Bangalore, paper, pp. 1-1, 8th - 10th December, 2003

[4]Hanaa. M. Said, Rania El Gohary, Mohamed Hamdy, Abdel-Badeeh “A Study on Data Mining Frameworks in Cyber Security" 2016

[5]Shiguo wang, "A Comprehensive Survey of Data Mining based Accounting-Fraud Detection Research", 2010 International Conference on Intelligent Computation Technology and Automation, IEEE, 2010

[6]K. Sathiyapriya and G. S. Sadasivam, “A survey on privacy preserving association rule mining,' Int. J. Data Mining Knowl. Manage. Process, vol. 3, no. 2, p. 119, 2013

[7]A. Miyaji and M. S. Rahman, "Privacy-preserving data mining: A game-theoretic approach,'” in Proc. 25th Data Appl. Security Privacy, 2011, pp. 186-200.

[8]S. Mitra, S. K. Pal, and P. Mitra, "Data mining in soft Computing framework: A survey", IEEE Trans. Neural Networks, vol. 13, pp. 3 - 14, 2006

[9]A. K. Kamrani and E. A. Nasr, "Data mining methodology and techniques," in Engineering Design and Rapid Prototyping Anonymous Springer, 2010

[10]M. S. Chen and J. H. Philip, "Data Mining: An Overview from a Database Perspective," IEEE Trans on knowledge and data engineering, vol. 8, no. 6, pp. 1-1, Dec 2013.

[11] Kumar, A., Vengatesan, K., Rajesh, M., \& Singhal, A. (2019). Teaching literacy through animation \& multimediaInt. J. Innovative Technol. Exploring Eng, 8(5), 73-76.

[12] Y. Xua, X. Qin, Z. Yang, Y. Yang, and K. Li, “A personalized k-anonymity privacy preserving method,' J. Inf. Computer. Sci., vol. 10, no. 1, pp. 139-155, 2013.

[13]M. N. Lakshmi and K. S. Rani, "SVD based data transformation methods for privacy preserving clustering,' 'Int. J. Computer. Appl., vol. 78, no. 3, pp. 39-43, 2013

[14].Sayyad, S., Mohammed, A., Shaga, V., Kumar, A., \& Vengatesan, K. (2018). Digital Marketing Framework Strategies Through Big Data. In International conference on Computer Networks, Big data and IoT (pp. 10651073). 